\title{
Active tuning of stroke-induced vibrations by tennis players
}

\section{Delphine Chadefaux, Guillaume Rao, Philippe Androuet, Eric Berton \& Laurent Vigouroux}

To cite this article: Delphine Chadefaux, Guillaume Rao, Philippe Androuet, Eric Berton \& Laurent Vigouroux (2017) Active tuning of stroke-induced vibrations by tennis players, Journal of Sports Sciences, 35:16, 1643-1651, DOI: 10.1080/02640414.2016.1227868

To link to this article: http://dx.doi.org/10.1080/02640414.2016.1227868

\section{Published online: 06 Sep 2016.}

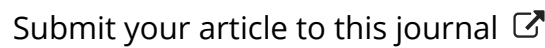

\section{Џ Article views: 40}

Q View related articles $\sqsubset$ 


\title{
Active tuning of stroke-induced vibrations by tennis players
}

\author{
Delphine Chadefaux ${ }^{\mathrm{a}}$, Guillaume Rao ${ }^{\mathrm{a}}$, Philippe Androuet ${ }^{\mathrm{b}}$, Eric Berton ${ }^{\mathrm{a}}$ and Laurent Vigouroux ${ }^{\mathrm{a}}$

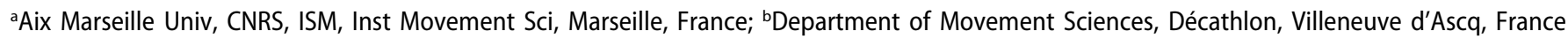

\section{ABSTRACT}

This paper investigates how tennis players control stroke-induced vibration. Its aim is to characterise how a tennis player deals with entering vibration waves or how he/she has the ability to finely adjust them. A specific experimental procedure was designed, based on simultaneously collecting sets of kinematic, vibration and electromyographic data during forehand strokes using various commercial rackets and stroke intensities. Using 14 expert players, a wide range of excitations at spectral and temporal levels were investigated. Energetic and spectral descriptors of stroke-induced vibration occurring at the racket handle and at the player's wrist and elbow were computed. Results indicated that vibrational characteristics are strongly governed by grip force and to a lower extent by the racket properties. Grip force management drives the amount of energy, as well as its distribution, into the forearm. Furthermore, hand-grip can be assimilated to an adaptive filter which can significantly modify the spectral parameters propagating into the player's upper limb. A significant outcome is that these spectral characteristics are as much dependent on the player as on the racket. This contribution opens up new perspectives in equipment manufacture by underlining the need to account for player/racket interaction in the design process.
ARTICLE HISTORY

Accepted 24 July 2016

\section{KEYWORDS}

Hand-tool interaction; grip force; shock propagation; high-resolution method; Tennis

\section{Introduction}

When playing tennis, stroke-induced vibration is directly transmitted to the body; the implication is that this is a key parameter governing performance and comfort (Stroede, Noble, \& Walker, 1999), and in preventing such conditions as tennis elbow (Brody, 1989; Hatze, 1976; Hennig, 2007; Wei, Chiang, Shiang, \& Chang, 2006).

Acknowledging that the characteristics of a player's grip can influence a racket's vibrational properties, several investigations focused on comparing the damping between hand-held, freely suspended and clamped tennis rackets. It was pointed out that hand-held racket vibration is more rapidly damped (about 10 times faster) than freely suspended racket vibration (Brody, 1989; Hatze, 1976). Cross (1997) has also shown that holding the racket results in a decrease of about $20 \%$ in the first bending mode frequency as compared with the free boundary conditions. This result is of primary importance since a correlation between the frequency of the racket's first bending mode, estimated under freely suspended conditions, and the amount of vibration transferred to the forearm following a ball impact has been pointed out (Hennig, 2007; Hennig, Rosenbaum, \& Milani, 1992). It follows that stroke-induced vibrations are not determined entirely by the mechanical properties of the racket, but rather that racket vibrations are also dependent on the playing velocity (Rogowski et al., 2015) and the attributes of the player's grip (Banwell, Roberts, Halkon, Rothberg, \& Mohr, 2014; Kawazoe \& Yoshinari, 2010).
This hypothesis is supported by the fact that, despite comparable grip forces, the magnitude of vibration transferred into the hands of skilled tennis players is significantly less than that of recreational players (Hennig, 2007). Likewise, less vibrational energy appears to be transmitted into these skilled players' forearms (Wei et al., 2006). As well as being dependent on the racket's mechanical properties, the available evidence suggests that stroke-induced vibrations are also dependent on the racket-hand interaction. However, no investigation has examined the extent to which the players themselves manage the transmission of vibration. Analysing the effect of handle size on the condition of tennis elbow, Rossi, Vigouroux, Barla, and Berton (2014) showed that players can finely adjust their grip force according to the handle size, suggesting that grip force is a means of driving the hand/racket interaction. Among the parameters under the players' control, we therefore hypothesised that, in addition to the control of the stroke performance, players' grip force adjustments might also be unconsciously used to tune the stroke-induced vibration transferred into their forearms, since this determines directly the mechanical linkage between the hand and the racket.

Assuming the hand-grip to be an adaptive filter, tuning the racket vibration transferred into the forearm therefore provides a means of protection from injury, as has already been demonstrated for the lower limb when running on various surfaces (Ferris, Liang, \& Farley, 1999). Consequently, developing an understanding of the hand/racket interaction could potentially allow sportsmen and racket manufacturers to exploit this relationship in preventing injury and/or enhancing 
performance. To address this question, an experiment was designed to collect stroke-induced vibrational parameters on both racket and forearm as well as grip forces over a wide range of inputs using various rackets and stroke intensities.

\section{Methods}

\subsection{Participants}

A total of 14 right-handed male participants with no noteworthy pathology ( $21 \pm 3$ years old, $182 \pm 8 \mathrm{~cm}$ tall, of weight $73 \pm 8 \mathrm{~kg}$ and right hand length $19.5 \pm 1.5 \mathrm{~cm}$ ) took part in the experiment. All participants were competitive tennis players (tennis practice: $11 \pm 4$ years, weekly training: $5 \pm 3 \mathrm{~h}$ ) and were classified according to the International Tennis Number (two ITN 2.0, three ITN 3.0, four ITN 5, four ITN 6.0 and one ITN 7.0). The experiment was approved by the local ethics committee and all the participants signed an informed consent form.

\subsection{Rackets}

Five commercially available rackets were used during the measurements (Table 1) and were set up with Artengo TA Feel strings stretched to $21 / 20 \mathrm{~kg}$. The rackets' grip size was fixed to $17.9 \%$ of the averaged hand length of the participants conveying to a $110 \mathrm{~mm}$ perimeter (Rossi, Berton, Grélot, Barla, \& Vigouroux, 2012). Note that the participants used to play with rackets from the tested brands, therefore it is assumed the mechanical characteristics of the tested rackets are in the range of participant own rackets. Modal analyses were carried out on each racket under free boundary conditions to extract bending, torsional and string-bed mode parameters, that is, shapes, eigenfrequencies $f_{k}^{0}$ and damping factors $\delta_{k}^{0}$ (Figure 1 (a)). A 22 point mesh distributed over the racket structure (17 points on the frame and 5 points on the string-bed) was used. The excitation force at each point on the mesh was provided by an impulse hammer (PCB Piezotronics; $\pm 400 \mathrm{~N} \mathrm{pk}$; resonant frequency $\geq 15 \mathrm{kHz}$ ).
Table 1. Mechanical characteristics of the rackets involved in the study.

\begin{tabular}{|c|c|c|c|c|c|}
\hline & $\begin{array}{l}\text { Wilson } \\
\text { Triad } 6 \\
\left(R_{1}\right)\end{array}$ & $\begin{array}{l}\text { Prince } \\
\mathrm{O} 3\left(\mathrm{R}_{2}\right)\end{array}$ & $\begin{array}{l}\text { Artengo } \\
\text { TR820 } \\
\left(\mathrm{R}_{3}\right)\end{array}$ & $\begin{array}{c}\text { Babolat } \\
\text { AeroPro } \\
\text { Drive }\left(\mathrm{R}_{4}\right)\end{array}$ & $\begin{array}{l}\text { Head } \\
\text { Prestige } \\
\left(\mathrm{R}_{5}\right)\end{array}$ \\
\hline Head surface $\left(\mathrm{m}^{2}\right)$ & 742 & 742 & 645 & 645 & 600 \\
\hline Mass $(\mathrm{g})$ & 253 & 259 & 282 & 304 & 327 \\
\hline Length $(\mathrm{cm})$ & 69.8 & 69.0 & 68.7 & 68.7 & 68.5 \\
\hline $\begin{array}{l}\text { Moment of inertia } \\
\left(\mathrm{kg} \cdot \mathrm{m}^{2}\right)\end{array}$ & 0.311 & 0.300 & 0.266 & 0.299 & 0.297 \\
\hline Static stiffness (Ra) & 36 & 71 & 76 & 73 & 64 \\
\hline $\begin{array}{l}\text { 1st bending mode } \\
\text { frequency }(\mathrm{Hz})\end{array}$ & 145.9 & 151.8 & 140.0 & 150.5 & 138.6 \\
\hline $\begin{array}{l}\text { 1st bending mode } \\
\text { damping factor } \\
(\%)\end{array}$ & 1.4 & 0.5 & 0.6 & 0.7 & 0.6 \\
\hline $\begin{array}{l}\text { 1st torsional mode } \\
\text { frequency }(\mathrm{Hz})\end{array}$ & 357.6 & 367.1 & 366.0 & 395.9 & 366.1 \\
\hline $\begin{array}{l}\text { 1st torsional mode } \\
\text { damping factor } \\
\text { (\%) }\end{array}$ & 1.4 & 0.6 & 1.2 & 0.7 & 1.4 \\
\hline
\end{tabular}

The rackets have been characterised with no string, no grip, and no measurement equipment. The moments of inertia were measured about the axis perpendicular to the plane of the racket through a pivot point $10 \mathrm{~cm}$ from the butt of the handle and that classical orders of magnitude have been obtained.

\subsection{Measurement protocol}

Before the measurement session, an unlimited warm-up period was allowed for familiarisation with the experimental conditions. The task consisted in hitting forehands with no ball spin toward a square-shaped target of sides $0.8 \mathrm{~m} \times 0.8 \mathrm{~m}$, $7 \mathrm{~m}$ away from the participant. The balls were fed using a throwing machine whose location was adjusted by the participants depending on their own assigned positions. The task was carried out under 15 conditions: the five rackets mentioned above combined with a range of three stroke intensities, referred to as "weak", "medium" and "hard" according to participants' own judgement. Participants were asked to make strokes which were as reproducible as possible. So as to allow participants to perform forehand strokes as close as possible to those they would make in an actual game, the point of impact of the ball on the strings was not fixed. For each trial, the kinematic properties of the stroke, grip position and the point of impact of the ball were visually checked to ensure
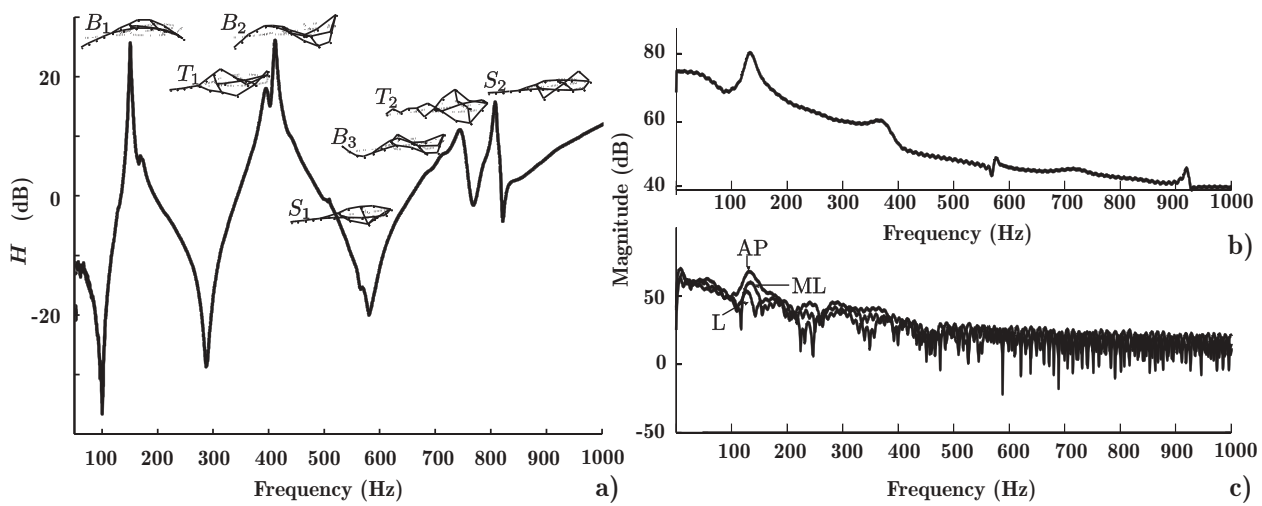

Figure 1. (a) Colocalised frequency response function measured on $R_{5}$ racket frame under free boundary conditions. Mode shapes are given at their corresponding modal frequencies. Bending, torsional and string-bed modes are recorded and labelled B, T and S respectively. Since the third string-bed mode frequency is greater than $1000 \mathrm{~Hz}$, it does not appear in the figure. Figure (b) and (c) are typical spectra of accelerometer signals measured on the racket handle and the participant's wrist, respectively. Observe the low-pass filter effect of the hand-grip with a cut-off frequency around $250 \mathrm{~Hz}$ at the wrist. These spectra were obtained during a "hard" forehand stroke. 
consistency in the processed database. Similarly, participants were instructed to maintain an identical grip technique and hand position from one racket to the next. Five targeted trials per task were collected. On average, $6.7 \pm 1.7,6.7 \pm 2.0$ and $7.1 \pm 2.6$ trials were required to achieve the five successful ones for "weak", "medium" and "hard" stroke intensities, respectively. The conditions were randomised (order of rackets and striking intensities) to avoid any effects due to learning or fatigue.

\subsection{Kinematics}

A high-resolution motion-capture system (Vicon Motion System, Oxford Metrics, UK) consisting of eight infrared cameras was used to capture the positions of 20 retro-reflective markers at $200 \mathrm{~Hz}$ (Figure 2). Nine markers were placed on the participants' trunk, upper arm and forearm, and five markers were fixed on the hand. Additionally, six markers were placed on the racket handle and head, and reflective tape was bonded onto the surface of the ball. These data were used to verify the consistency in the stroke kinematics and in the

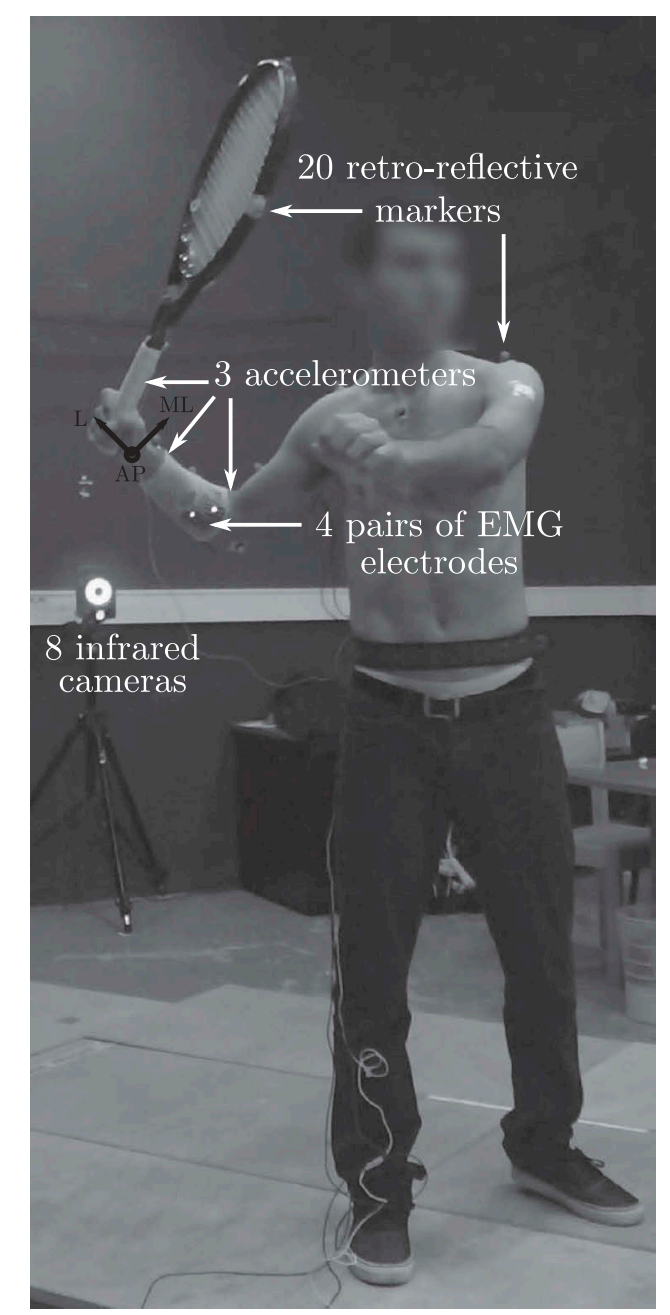

Figure 2. Picture of a participant taken during the experimental test. Each participant was fitted with 2 accelerometers, 20 markers for kinematic measurements and 4 pairs of surface electromyographic (EMG) electrodes. One accelerometer and 6 markers were placed on the racket. grip position on the racket handle, as well as for estimating the point of impact of the ball on the stringing.

\subsection{Ball impact intensity}

The intensity of the ball impact is derived from the definition of the transfer function between the excitation force $F_{i}$ provided by an impact on the racket's string-bed and the resulting normal acceleration $S$; this transfer function is defined as

$$
H_{i}(\omega)=\frac{S(\omega)}{F_{i}(\omega)},
$$

where $\omega=2 \pi f$ is the angular frequency, and suffix $i$ refers to the location at which the impact occurred on the string-bed. $S$ was collected directly during the measurement sessions and based on a single-axis accelerometer (Dytran; $\pm 500 \mathrm{~g} \mathrm{pk}$; [1.6-10,000] Hz) bonded onto the racket handle $15 \mathrm{~cm}$ from the butt cap. For each racket, the $H_{i}$ was determined in the modal analysis at five impact locations $i$ on the string-bed. Hence, for a given ball impact at a location $j$ determined from kinematic data, we selected the transfer function $H_{i}$ with the closest $i$ to $j$ in the five point mesh and computed $F_{j}(\omega)=S(\omega) / H_{i}(\omega)$. Finally, to facilitate interpretation, a dimensionless indicator of ball impact intensity $F_{b}$ was defined for each participant by dividing the entire set of estimated excitation forces by the maximum value obtained over the entire experiment.

\subsection{Grip force}

Grip force measurement when gripping a handle remains a scientific challenge (Rossi et al., 2012; Wu, Dong, Warren, Welcome, \& McDowell, 2014). This could be accomplished by instrumenting the handle with strain gauges or a pressure mapping sensor. However, as these methods affect the hand/racket interface, they were unsuitable for this study. We therefore chose to compute a grip force index based on existing methods that estimate grip force based on electromyographic (EMG) data (Duque, Masset, \& Malchaire, 1995; Hoozemans \& Van Dieën, 2005; Roman-Liu \& Tokarski, 2002). EMG signals from four forearm muscles (the extensor digitorum communis, the extensor carpi radialis longus, the flexor digitorum profondus and superficialis and the flexor carpi ulnaris) were collected using a wireless system (Delsys Trigno, Natick, MA, USA, $F_{s}=1925 \mathrm{~Hz}$ ). Electrodes were positioned after appropriate skin preparation on the bellies of the respective muscles. The placement of the electrodes was determined using anatomical descriptions, palpation and functional movements, and monitored by recording the signals during functional tests. A calibration task was performed at the start of the measurement session. When seated, participants were asked to apply several patterns of grip force with the right hand on an instrumented cylindrical handle whose diameter closely approximated that of the rackets used (Handle dynamometer, Sixaxes, Argenteuil, France) (Rossi et al., 2012). The patterns involved a maximal voluntary contraction (MVC) task, followed by a stepped force increase until the MVC value was reached, and a sinusoidal fluctuating grip 
task (Hoozemans \& Van Dieën, 2005). EMG signals passed through a bandpass filter and full-wave rectifier with zero phase shift ([20-400] Hz; 4th order Butterworth). The associated root mean square signals were then calculated using a 500 ms moving window (Rossi et al., 2014; Valero-Cuevas, Zajac, \& Burgar, 1998). The final grip force index, referred to as $\mathrm{F}_{g}$, was determined by normalising the predicted grip force by the maximum value obtained for each participant over the entire experiment.

\subsection{Vibrational energy}

Two 3-axis accelerometers (Endevco; $\pm 50 \mathrm{~g}$ pk; [20-6000] Hz) were placed at the midpoint between the radial and ulnar styloid processes and at the lateral epicondyle of the humerus. The normal acceleration of the racket was measured using a single-axis accelerometer (Dytran; $\pm 500 \mathrm{~g} \mathrm{pk}$; $[1.6-10,000] \mathrm{Hz}$ ) bonded onto the racket handle $15 \mathrm{~cm}$ from the butt cap. The entire set of accelerometer signals was recorded synchronously through the Vicon data acquisition card at $12,000 \mathrm{~Hz}$. Raw signals were passed through a band-pass filter with zero phase shift ([20-1000] Hz for the joints and [20-1500] Hz for the racket; 4th order Butterworth). The lower limit of the filters was aimed at removing motion artefacts and the upper limits of the filters were selected according to the human skin sensitivity to vibration (Griffin, 1990) and the tennis racket vibration content (Stroede et al., 1999). Then, filtered signals were segmented from the onset of the impact. The "onset of impact" was determined as the instant at which the racket's normal acceleration reached its maximum value.

The total vibrational energy reaching the wrist $\left(E_{w}\right)$ and the elbow $\left(E_{e}\right)$, as well as vibrational energy at the wrist in mediolateral $\left(E_{w}^{M L}\right)$, anteroposterior $\left(E_{w}^{A P}\right)$ and longitudinal axes $\left(E_{w}^{L}\right)$ were computed in accordance with classical signal processing.

$$
E=\frac{1}{N_{t}} \sum_{n=1}^{N_{t}}\left|s_{n}\right|^{2}
$$

where $E$ is the energy of a given discrete signal $s_{n}$, and $N_{t}=$ $T F_{s}$, where $T$ is a $100 \mathrm{~ms}$ window from the impact onset and $F_{S}$ the sampling rate. To estimate the percentage of vibrational energy transferred into the forearm, the ratio $R_{f}=E_{e} / E_{w}$ was defined. Moreover, to gain insight into the spatial distribution of vibrational energy at the wrist, the ratios $R_{M L}=E_{w}^{M L} / E_{w}$, $R_{A P}=E_{w}^{A P} / E_{w}$ and $R_{L}=E_{w}^{L} / E_{w}$ were defined.

\subsection{Spectral content}

To investigate the spectral content of stroke-induced vibration, the cut-off frequencies of vibration signals reaching the participants' wrist $f_{c}^{M L}, f_{c}^{A P}$ and $f_{c}^{L}$ were firstly determined. They were defined as those frequencies for which only $1 \%$ of the energy remains in the spectrum. To this end, the spectral energy was computed for each spectrum of the accelerometer signals collected on the participants' wrist based on Equation 2.

Second, modal parameters were extracted from the accelerometer measurements made on the rackets. It was assumed that the collected signals could be described by the noiseless Exponential Sinusoidal Model

$$
s(t)=\sum_{k=1}^{K} a_{k} \cos \left(2 \pi f_{k} t+\phi_{k}\right) e^{-2 \pi f_{k} \delta_{k} t},
$$

where suffix $k$ indicates the components required to properly describe the signal, $\mathrm{K}$ is the model order, $t$ is the dimensionless time vector, $f_{k} \in\left[-\frac{1}{2} ; \frac{1}{2}\right]$ is the frequency of the $k$ th component, $a_{k}$ is the positive amplitude, $\delta_{k}$ is the damping factor and $\phi_{k} \in$ $[-\pi ; \pi]$ is the phase. A high-resolution method (ESPRIT: estimation of signal parameters via rotational invariance techniques) was then used to accurately estimate the modal parameters $\left(f_{k}, \delta_{k}\right.$ $a_{k}$ and $\Phi_{k}$ ) in each recorded signal (Roy, Paulraj, \& Lailath, 1986). Investigating the modal parameter $a_{k}$ is not relevant because of the lack of accuracy in detecting the point of impact of the ball. Therefore, variations in parameters $f_{k}$ and $\delta_{k}$ were investigated via the descriptors $\Delta f_{k}=\frac{f_{k}-f_{k}^{0}}{f_{k}^{0}}$ and $\Delta \delta_{k}=\frac{\delta_{k}-\delta_{k}^{0}}{\delta_{k}^{0}}$, where $f_{k}^{0}$ and $\delta_{k}^{0}$ are the eigenfrequencies and the damping factors estimated under the free boundary conditions as given in Table 1.

\subsection{Statistical analysis}

For each participant, mean values of each defined variable were computed over the five trials collected for each condition. Two-way-repeated analysis of variance measurements with Holm-Bonferroni correction were carried out to highlight the effect of stroke intensity and racket conditions on the ball impact intensity $\left(F_{b}\right)$, the participant's grip force $\left(F_{g}\right)$ and the characteristics of stroke-induced vibration $\left(E_{w}, E_{e}, R_{f}, R_{M L}, R_{A P}\right.$ $R_{L}, f_{c}^{M L}, f_{c}^{A P}, f_{c}^{L}, \Delta f_{k}$ and $\left.\Delta \delta_{k}\right)$. When a significant effect was observed ( $p<0.05)$, a Bonferroni post hoc test was carried out to determine significant differences. Pearson correlations were calculated to identify the relationships between the mechanical properties of the rackets and the characteristics of the stroke-induced vibration.

\section{Results}

\subsection{Ball impact intensity and grip force}

The participants performed forehand with ball impact intensity according to the stroke intensity condition $(F(2,209)=77.0$; $p<0.01)$, and with no difference from one racket to the next (see Figure 3(a)). Concomitantly, the stroke intensity and the racket conditions had a significant main effect on the grip force index $(F(2,209)=57.7 ; p<0.01$ and $F(4,209)=6.7 ; p<0.01$, respectively). Figure 4 indicates that the grip force index increased with the stroke intensity. On average, "weak", "medium" and "hard" stroke intensities corresponded to averaged grip force index values of $44 \pm 19 \%, 56 \pm 19 \%$ and $77 \pm 19 \%$, respectively. Besides, the grip force index was lower for the rackets $R_{2,3,4}$ (54 $\pm 23 \%)$ than the $R_{5}(70 \pm 22 \%)$.

\subsection{Energetic aspects}

Results obtained for the vibrational energies (Figure 3(b) and (c)) show that an increase in the ball impact intensity implied 

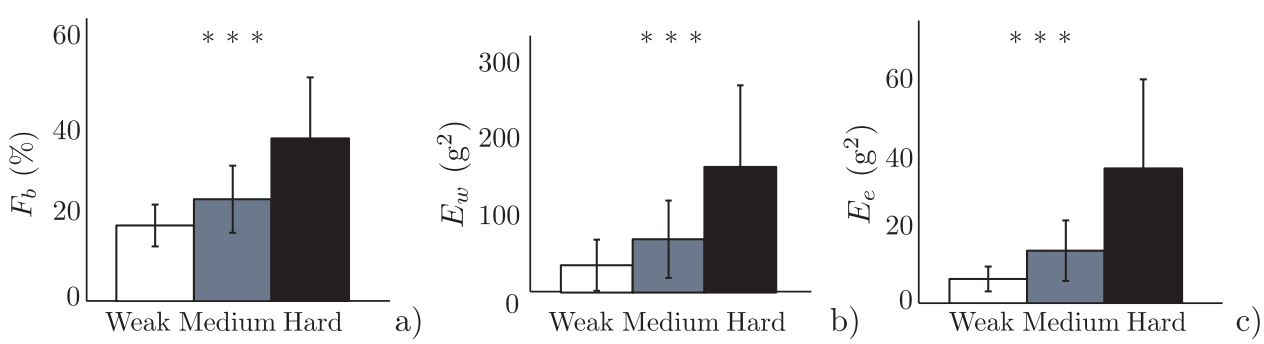

Figure 3. (a) Evolution of the ball impact intensity $\left(F_{b}\right)$ according to the three stroke intensity conditions. Figure (b) and (c) shows changes in vibrational energy transmitted to the wrist $\left(E_{w}\right)$ and to the elbow $\left(E_{e}\right)$ according to the three stroke intensity conditions. $* * *$ indicates significant differences $(p<0.05)$ between all the stroke intensity conditions.

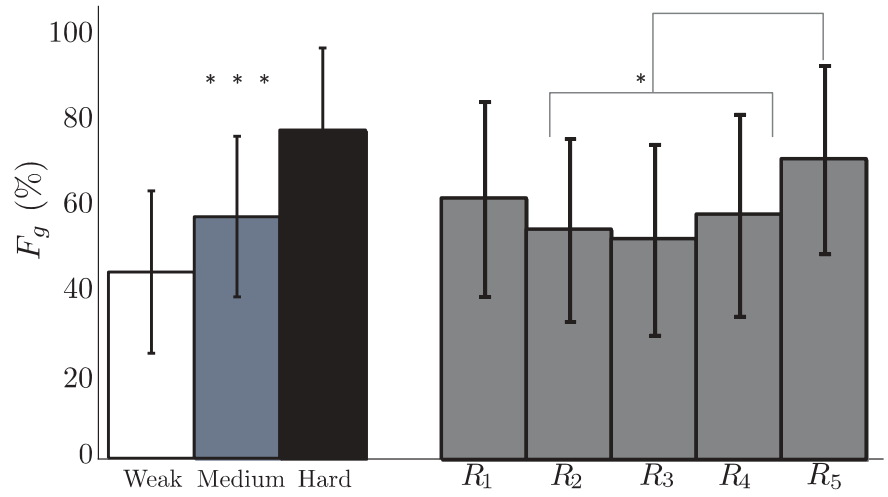

Figure 4. Changes in grip force intensity $\left(F_{g}\right)$ according to the five rackets and the three stroke intensity conditions. $*$ indicates significant difference $(p<0.05)$ between two racket conditions. $* * *$ indicates significant differences $(p<0.05)$ between all the stroke intensity conditions.

an increase in the vibrational energy transmitted to the wrist $(F(2,209)=59.5 ; p<0.01)$ and the elbow $(F(2,209)=74.0$; $p<0.01)$. The vibrational energies transmitted to the wrist and the elbow were correlated with the grip force index $(R=0.34 ; p<0.01$, and $R=0.44 ; p<0.01)$, and increased by $562 \pm 424 \%$ and $617 \pm 453 \%$ between "weak" and "hard" stroke intensity conditions, respectively. Additionally, it should be noted that the vibrational energy transmitted to the wrist was also driven by the dynamic behaviour of the racket: the lower the first bending mode frequency $f_{B 1}$, the higher the vibrational energy transmitted to the wrist $(R=-0.94$; $p=0.02$ )

On average, the proportion of vibrational energy transmitted to the wrist reaching the elbow was estimated as $R_{f}=28 \pm 19 \%$, with $R_{f}$ varying from $11 \%$ to $87 \%$ (see Figure 5(a)). Further, the vibrational energy reaching the wrist and the elbow was linearly correlated for each participant independently $\left(R^{2}=0.91 \pm 0.06\right)$.

Focusing on the vibrational energy transmitted to the wrist shows that $51 \pm 11 \%$ was concentrated along the anteroposterior axis, i.e., the ball impact direction, while $25 \pm 10 \%$ and $23 \pm 10 \%$ were concentrated along the mediolateral and the longitudinal axes, respectively (see Figure 5(b)-(d)). Interestingly, the ratios of vibrational energy at the wrist were significantly related to the stroke intensity $(F(2,209)=14.3 ; p<0.01, F$ $(2,209)=3.4 ; p=0.04$, and $F(2,209)=44.6 ; p<0.01$, for the mediolateral, anteroposterior and longitudinal directions, respectively). These ratios decreased from $55 \pm 10 \%$ and $28 \pm 11 \%$ to $46 \pm 10 \%$ and $24 \pm 8 \%$ between "weak" and "hard" stroke

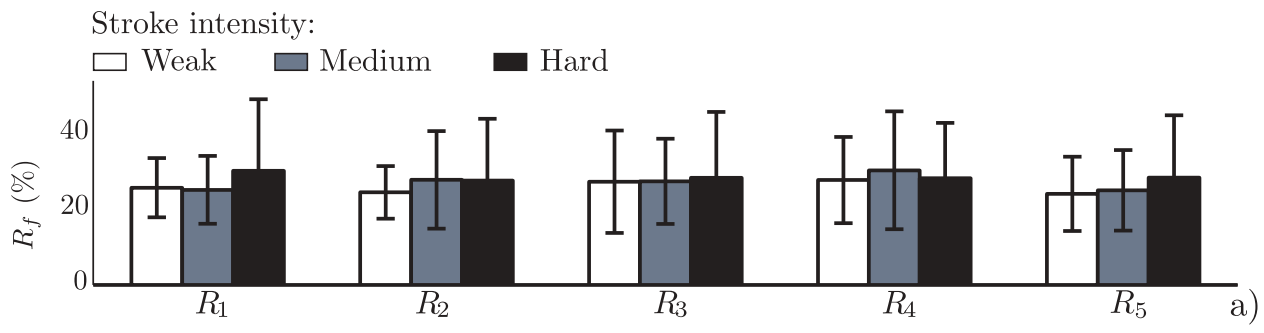

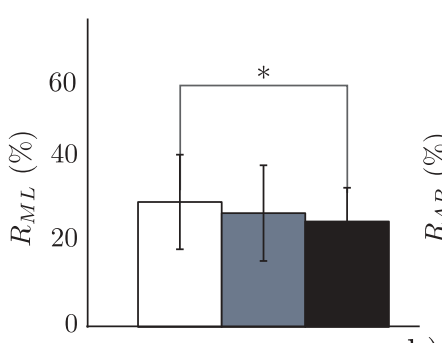

b)

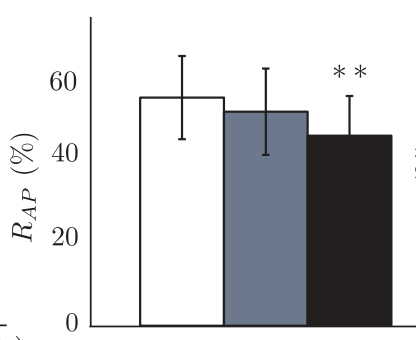

c)

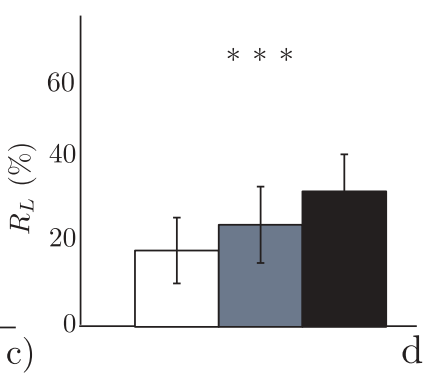

d)

Figure 5. (a) Percentage of vibrational energy transferred into the forearm $\left(R_{f}\right)$ for the five rackets under the three stroke intensity conditions. Figure (b), (c) and (d) shows the spatial distribution of vibrational energy at the wrist along the mediolateral $\left(R_{M L}\right)$, anteroposterior $\left(R_{A P}\right)$ and longitudinal $\left(R_{L}\right)$ axes according to the three stroke intensity conditions. $*$ indicates significant difference $(p<0.05)$ between two stroke intensity conditions. $* *$ indicates significant differences $(p<0.05)$ with the two other stroke intensity conditions. $* * *$ indicates significant differences $(p<0.05)$ between stroke intensity conditions. 
intensity conditions along the anteroposterior and mediolateral axes, respectively, while it increased from $17 \pm 8 \%$ to $31 \pm 8 \%$ along the longitudinal axis. Moreover, the grip force index was also correlated with the distribution of vibrational energy at the wrist $(R=-0.22 ; p<0.01, R=-0.15 ; p=0.02$ and $R=0.39$; $p<0.01$, for the anteroposterior, the mediolateral and the longitudinal axes, respectively).

\subsection{Spectral content}

Typical spectra of accelerometer signals as measured on the racket handle and the participant's wrist are shown in Figure 1(b) and (c). A significant observation is that the impact of the ball generated spectral content up to $1500 \mathrm{~Hz}$ for the racket. Regarding the wrist, the energy propagating from the racket was strongly filtered: the cut-off frequencies measured in the three directions were around $250 \mathrm{~Hz}$. Interactions between the stroke intensity and the racket conditions were observed $(F(8,209)=4.0 ; p<0.01, F(8,209)=9.0$; $p<0.01$, and $F(8,209)=7.7 ; p<0.01$ for the mediolateral, anteroposterior and longitudinal axes, respectively). Figure 6 indicates that the cut-off frequencies tended to increase with stroke intensity in various extent according to the racket and the axis. For instance, considering the longitudinal axis, the cut-off frequency decreased by $27 \pm 21 \mathrm{~Hz}$ for the racket $R_{1}$, while it increased by $25 \pm 11 \mathrm{~Hz}$ for the racket $R_{2}$, and followed an inverted U-shaped curve $(+27 \pm 21 \mathrm{~Hz}$ and $-18 \pm 15 \mathrm{~Hz})$ for the racket $R_{4}$. Based on the classical modal analyses carried out on the rackets, these results show that only the first bending mode was transmitted into the participant's forearm. In accordance with the results given in Section 3.2, the amplitude measured at the first bending mode frequency $f_{B 1}$ depended on the direction, with a higher peak being observed along the anteroposterior axis than along the mediolateral and longitudinal directions (see Figure 1(b) and (c)).

Based on the foregoing, variations in frequency and damping factor of the racket's first bending mode were analysed. Interactions between stroke intensity and racket conditions were observed $(F(8,209)=6.1 ; p<0.01, F$ $(8,209)=3.0 ; p<0.01$, for variations in frequency and damping factor of the racket's first bending mode, respectively). Over all the conditions investigated, $f_{B 1}$ decreased by $2-13 \%$ and $\delta_{B 1}$ increased by $403-1819 \%$ when the participant holds the racket. Figure 7 shows that while $f_{B 1}$ tended to decrease as stroke intensity increases, no obvious trend was observed for $\delta_{B 1}$. Investigating further how these variations in the first bending mode characteristics were related to the mechanical properties of the racket, variations in the racket's first bending mode frequency were related to the racket length $(R=-0.92 ; p=0.03)$ and static stiffness $(R=0.95 ; p=0.01)$ while variations in the racket's first bending mode damping factor were related to the first torsional mode frequency $(R=-0.99 ; p<0.01)$.

Figure 8 shows the mean values of $f_{B 1}$ and $\delta_{B 1}$ over all the investigated conditions as compared with the associated free boundary conditions $f_{B 1}^{0}$ and $\delta_{B 1}^{0}$. A noticeable result is that the player/racket coupling affected the dynamic behaviour of the racket as least as much as its mechanical parameters. For instance, when held, the racket $R_{2}$ showed modal parameters closer to the racket $R_{1}$ than to its own parameters under free boundary conditions.

Stroke intensity:

$\square$ Weak $\square$ Medium $\square$ Hard
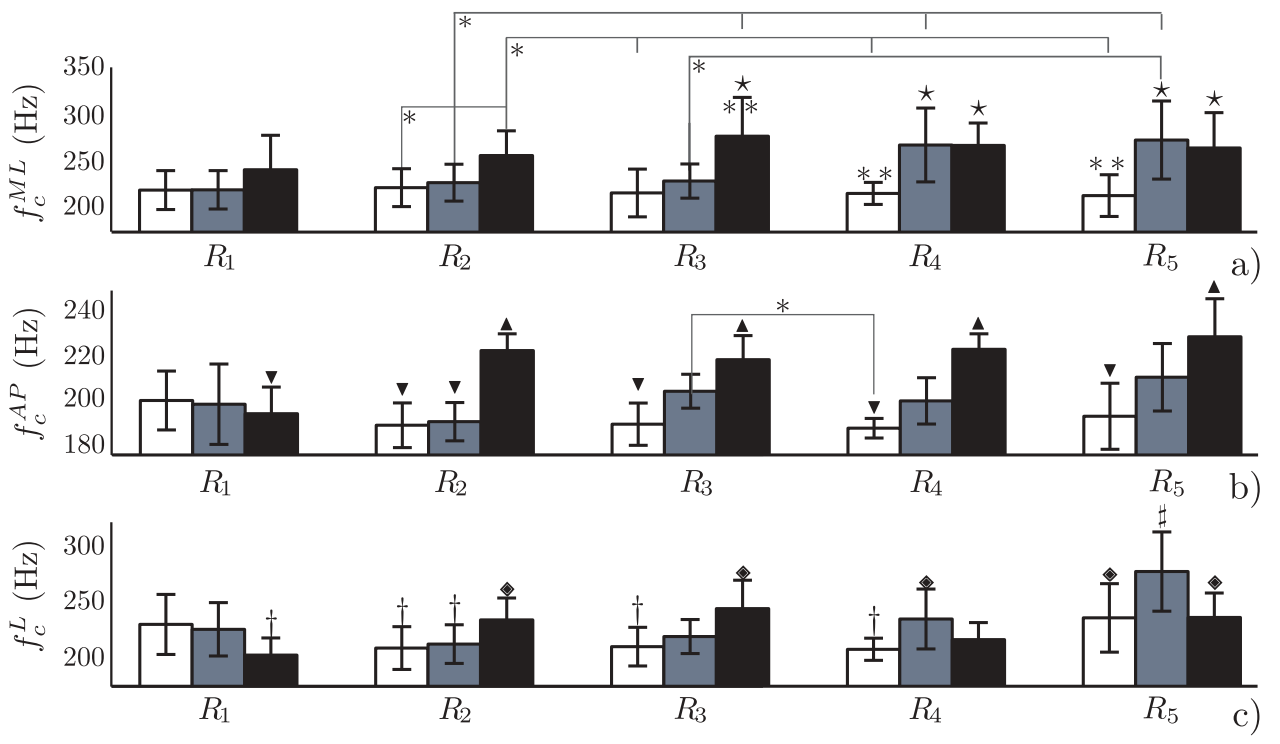

Figure 6. Cut-off frequencies estimated along the mediolateral $\left(f_{c}^{M L}\right)(a)$, anteroposterior $\left(f_{c}^{A P}\right)(b)$ and longitudinal $\left(f_{c}^{L}\right)(c)$ axes for the five rackets under the three stroke intensity conditions. $*$ indicates significant difference $(p<0.05)$ between two conditions. $* *$ indicates significant differences $(p<0.05)$ with the two other stroke intensity conditions under the same racket condition. $\star$ indicates significant difference $(p<0.05)$ with all weak stroke intensity conditions as well as with $R_{1}$ in medium stroke intensity condition. $\boldsymbol{\nabla}$ indicates significant difference $(p<0.05)$ with the $R_{5}$ racket condition in medium stroke intensity condition. $\mathbf{\Delta}$ indicates significant difference $(p<0.05)$ with the $R_{2,3,4,5}$ racket conditions in weak stroke intensity condition, as well as with the $R_{1,2,3,4}$ racket conditions in medium stroke intensity condition and with the $R_{1}$ racket condition in hard stroke intensity condition. $\dagger$ indicates significant difference $(p<0.05)$ with the $R_{3}$ racket condition in hard stroke intensity condition. indicates significant difference $(p<0.05)$ with the $R_{1}$ racket condition in hard stroke intensity condition. \# indicates significant difference $(p<0.05)$ with all other conditions. 


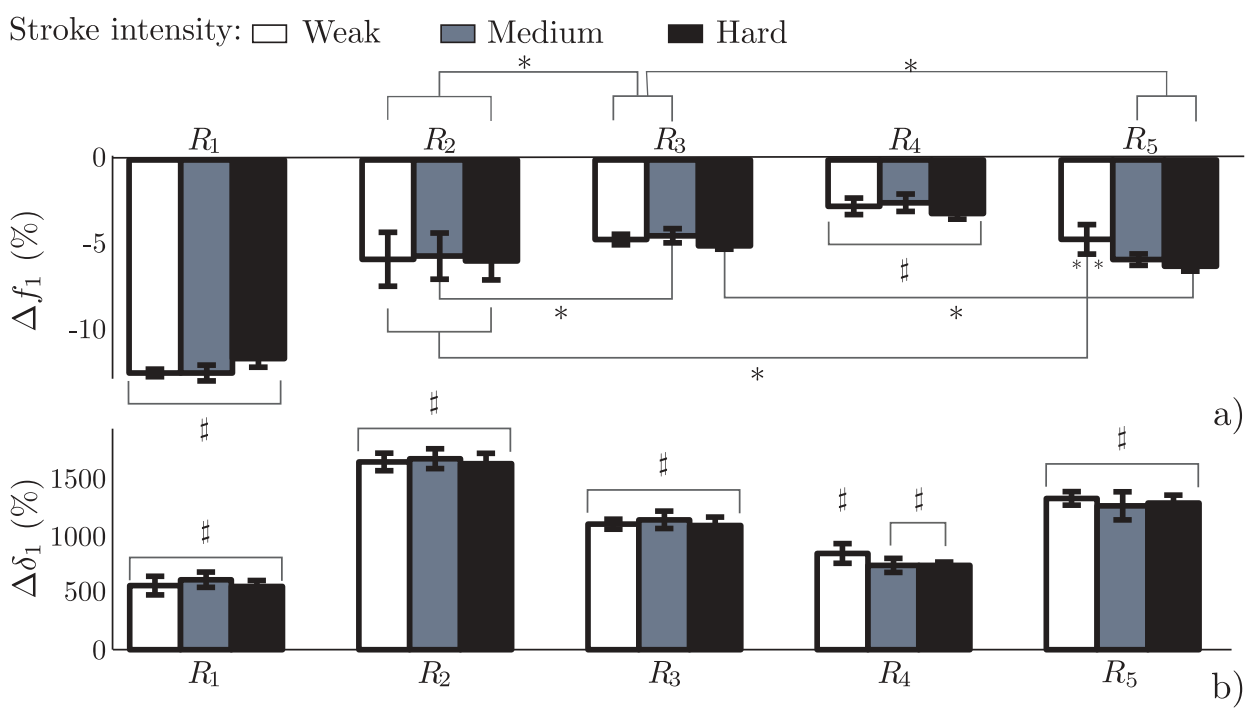

Figure 7. Variations in frequency $\left(\Delta f_{B 1}\right)$ and damping factor $\left(\Delta \delta_{B 1}\right)$ of the racket's first bending mode for the five rackets under the three stroke intensity conditions. $*$ indicates significant difference $(p<0.05)$ between two conditions. $* *$ indicates significant differences $(p<0.05)$ with the two other stroke intensity conditions under the same racket condition. \# indicates significant differences $(p<0.05)$ with all other conditions.

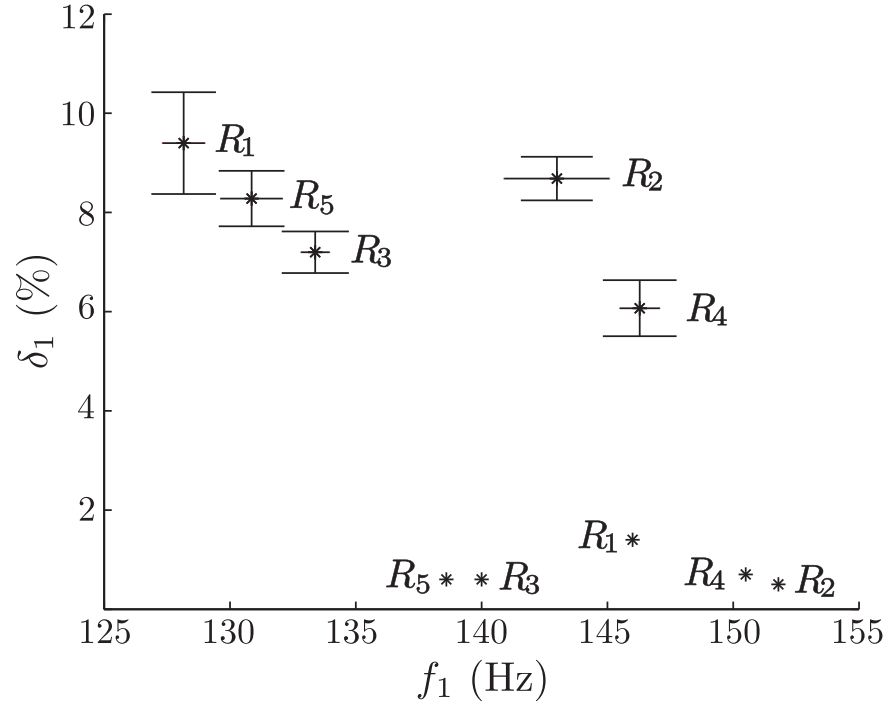

Figure 8. Changes in the first bending mode parameters (frequency $f_{1}$ and damping factor $\delta_{1}$ ) of the five rackets over all the stroke intensity conditions investigated with respect to their values under free boundary conditions (frequency $f_{1}^{0}$ and damping factor $\delta_{1}^{0}$ ). Observe that $f_{1}$ and $\delta_{1}$ of a hand-held racket can become closer to $f_{1}^{0}$ and $\delta_{1}^{0}$ of another racket than to its own $f_{1}^{0}$ and $\delta_{1}^{0}$. For example, when hand-held, $R_{1}$ is closer to $R_{5}$ under free boundary conditions than it is to itself under these same free boundary conditions.

\section{Discussion}

This study investigated the ability of the tennis player to finely control (voluntarily or not) stroke-induced vibration in tennis performance. This issue was addressed over a wide range of vibrational inputs by varying both stroke intensity and racket. A noteworthy observation is that the vibrational content is modified to a remarkable degree by the grip force according to the stroke intensity and the racket. These observations confirm that the hand-grip can be considered as an adaptive filter driven in particular by grip force to tune the vibration entering the forearm from both energetic and spectral points of view.

From an energetic point of view, as indicated by Rogowski et al. (2015), the harder the stroke, the greater the vibrational energy transferred into the forearm. Specifically, our results show that vibrational energy is mostly concentrated along the ball impact axis, regardless of the stroke intensity and the racket properties. However, there is an increase in the amount of vibrational energy transmitted along the longitudinal axis when the stroke intensity increases. As the grip force increases with the stroke intensity, the mechanical coupling is then reinforced at the hand/racket interface, limiting disturbing movements along the impact axis and favouring the successful completion of the stroke. This behaviour may be understood as a performance/protection process as it stabilises the conditions for execution of the movement. Moreover, akin to observations made by Hennig et al. (1992) and Wei et al. (2006), our results highlighted a constant ratio of vibrational energy propagating from the wrist to the elbow averaging $28 \pm 19 \%$. Hennig et al. (1992) have investigated strokeinduced vibration in 24 participants at various levels of expertise using 23 rackets. They suggested that the vibrational energy at the elbow is less than $26 \%$ of the wrist value. Wei et al. (2006) analysed backhands performed by 12 skilled and 12 amateur players using their own rackets. Peak acceleration ratios between wrist and elbow were $28 \%$ for skilled and $60 \%$ for amateur players. Our outcomes corroborate hence these studies by suggesting that the ratio of vibrational energy propagating from the wrist to the elbow is controlled regardless of the stroke intensity and racket properties, though it is specific to each participant. An assumption related to this fact is that keeping constant the energy ratio transmitted from the wrist to the elbow may act as a protective mechanism for the musculoskeletal system and limits the transmission of vibrational energy to the anatomical structures of the elbow and the arm. However, since the ratio of vibrational energy propagating from the wrist to the elbow is mostly driven by grip 
force, its minimisation requires a compromise between ball speed and accuracy, which explains why this ratio reaches relatively high values for several participants. We therefore assume that raising awareness among tennis players on the importance of grip force in terms of vibration energy transmission would prevent situations which might lead to injury, as exposure to vibration is known to be related to various kinds of injury (e.g., Raynaud syndrome, carpal tunnel syndrome and tendonitis) (Griffin, 1990).

From a spectral point of view, as already observed by Hennig (2007), our findings indicated that a higher racket first bending mode frequency implies less energy propagating into the wrist. Further investigation of the spectral domain shows that the frequency range observable at the wrist increases with stroke intensity. The hand/racket coupling filters energy above $250 \mathrm{~Hz}$, which could be related to the $500 \mathrm{~Hz}$ upper limit of human skin sensitivity (Goff, 1967) and the $100-320 \mathrm{~Hz}$ range of high sensitivity to mechanical vibration. Additionally, the first bending mode parameters are affected by the hand/racket coupling depending both on stroke intensity and racket properties. In all configurations, the frequency decreases and the damping factor significantly increases as compared with their values under free boundary conditions. A substantial knowledge brought out by the present study is that these variations are not constant for a given player, but mostly depend on the stroke intensity and the racket properties. The first bending mode frequency deviates more from its value under free boundary conditions for a higher stroke intensity, and for a longer but less stiff racket, while the first bending mode damping factor deviates more from its value under free boundary conditions for a racket with a high first torsional mode frequency. Analysing how the presence of the player's hand affects the dynamic behaviour of the tennis racket, Brody $(1987,1989)$ pointed out an increase in the temporal decay rate relative to the freely suspended conditions. Based on spectral investigations, Banwell et al. (2014) also identified frequency deviations and damping factor changes up to $1500 \mathrm{~Hz}$. Concerning analyses under actual playing conditions, Hennig et al. (1992) indicated that less experienced players seem to generate slightly more frequency deviation in the first bending mode than expert players. In accordance with these results, we also showed that players possess the ability to tune the racket's dynamic behaviour to such an extent that the first bending mode parameters of a hand-held racket can become closer to those of another racket than to those of its own under free boundary conditions.

Examining our findings, it should be considered that the main limitation lies in gathering accurate measurements of the stroke performance while preserving the player/racket interface. Especially, the lack of information about the ball impact led us to analyse the total amount of energy transmitted into the participants' forearms rather than the energy of each excited mode. Besides, the grip force computations were based on indirect estimations based on EMG measurements preventing the highlighting of the actual value. Even if these indirect methods allowed us to make comparison in between our experimental conditions, they could lead to inaccuracies in physical value measurement. Complementary studies would therefore be valuable to validate and develop dedicated methods to solve this experimental lock.

\section{Conclusion}

The main contribution of this study is that hand-grip during tennis stroke can be understood as an adaptive filter able to adjust the vibration entering the forearm. Our results indicated that the tennis player is able to tune, voluntarily or not, the stroke-induced vibration at both spectral content and energy distribution levels. Tennis instructors and racket manufacturers could then help tennis players reach the desired "feel" through optimising their grip force, as well as in the choice and the design of rackets of suitable dynamic behaviour. Finally, although no previous study has described the spatial distribution of the vibrational energy at the player's forearm, it appears critical in understanding the control of stroke-induced vibration by tennis players. This will be of great value in enhancing tennis racket design with respect to the significant player/racket interactions during play, while trying to reduce injury.

\section{Acknowledgements}

The authors would like to thank Chris Hayot for his involvement in designing the experimental procedure and in the measurements; Vivien Cabos, Jordan Lecoeuvre and Alina Tolmunen for their help during the measurements and the tennis players who participated in this study.

\section{Disclosure statement}

The authors disclose professional relationships with companies or manufacturers who will benefit from the results of the present study. The results of this study do not constitute endorsement by ACSM.

\section{References}

Banwell, G. H., Roberts, J. R., Halkon, B. J., Rothberg, S. J., \& Mohr, S. (2014) Understanding the dynamic behaviour of a tennis racket under play conditions. Experimental Mechanics, 54, 527-537. doi:10.1007/s11340013-9803-9

Brody, H. (1987). Models of tennis racket impacts. International Journal of Sport Biomechanics, 3, 293-296. doi:10.1123/ijsb.3.3.293

Brody, H. (1989). Vibration damping of tennis rackets. International Journal of Sport Biomechanics, 5, 451-456. doi:10.1123/ijsb.5.4.451

Cross, R. (1997). The dead spot of a tennis racket. American Journal of Physics, 65(8), 754-764. doi:10.1119/1.18646

Duque, J., Masset, D., \& Malchaire, J. (1995). Evaluation of handgrip force from EMG measurements. Applied Ergonomics, 26(1), 61-66. doi:10.1016/ 0003-6870(94)00003-H

Ferris, D. P., Liang, K., \& Farley, C. T. (1999). Runners adjust leg stiffness for their first step on a new running surface. Journal of Biomechanics, 32, 787-794. doi:10.1016/S0021-9290(99)00078-0

Goff, G. D. (1967). Differential discrimination of frequency of cutaneous mechanical vibration. Journal of Experimental Psychology, 74, 294-299. doi:10.1037/h0024561

Griffin, M. J. (1990). Handbook of human vibration. London: Academic Press Edition. ISBN 0123030412.

Hatze, H. (1976). Forces and duration of impact, and grip tightness during the tennis stroke. Medicine \& Science in Sports \& Exercise, 8, 88-95. doi:10.1249/00005768-197600820-00014

Hennig, E. M. (2007). Influence of racket properties on injuries and performance in tennis. Exercise and Sport Sciences Reviews, 35(2), 62-66. 
Hennig, E. M., Rosenbaum, D., \& Milani, T. L. (1992). Transfer of tennis racket vibrations onto the human forearm. Medicine \& Science in Sports \& Exercise, 24(10), 1134-1140. doi:10.1249/00005768-199210000-00011

Hoozemans, M. J. M., \& Van Dieën, J. H. (2005). Prediction of handgrip forces using surface EMG of forearm muscles. Journal of Electromyography and Kinesiology, 15, 358-366. doi:10.1016/j. jelekin.2004.09.001

Kawazoe, Y., \& Yoshinari, K. (2010). Prediction of the impact shock vibrations of the tennis player's wrist joint: Comparison between two super large sized tennis rackets with different frame weight distributions. Journal of System Design and Dynamics, 4(2), 331-347. doi:10.1299/jsdd.4.331

Rogowski, I., Creveaux, T., Triquigneaux, S., Macé, P., Gauthier, F., \& Sevrez, V. (2015). Tennis racket vibrations and shock transmission to the wrist during forehand drive. PLOS ONE, 10(7), 1-10. doi:10.1371/journal. pone.0132925

Roman-Liu, D., \& Tokarski, T. (2002). EMG of arm and forearm muscle activities with regard to handgrip force in relation to upper limb location. Acta of Bioengineering and Biomechanics, 4(2), 33-47.

Rossi, J., Berton, E., Grélot, L., Barla, C., \& Vigouroux, L. (2012). Characterisation of forces exerted by the entire hand during the power grip: Effect of the handle diameter. Ergonomics, 55, 682-692. doi:10.1080/00140139.2011.652195

Rossi, J., Vigouroux, L., Barla, C., \& Berton, E. (2014). Potential effects of racket grip size on lateral epicondilalgy risks. Scandinavian
Journal of Medicine \& Science in Sports, 24, 462-470. doi:10.1111/ sms.12204

Roy, R., Paulraj, A., \& Lailath, T. (1986). ESPRIT - A subspace rotation approach to estimation of parameters of cisoids in noise. IEEE Transactions on Acoustics, Speech, and Signal Processing. doi:10.1109/ TASSP.1986.1164935

Stroede, C. L., Noble, L., \& Walker, H. S. (1999). The effect of tennis racket string vibration dampers on racket handle vibrations and discomfort following impacts. Journal of Sports Sciences, 17, 379-385. doi:10.1080/ 026404199365894

Valero-Cuevas, F. J., Zajac, F. E., \& Burgar, C. G. (1998). Large index-fingertip forces are produced by subject-independent patterns of muscle excitation. Journal of Biomechanics, 31, 693-703. doi:10.1016/S0021-9290(98) 00082-7

Wei, S. H., Chiang, J. Y., Shiang, T. Y., \& Chang, H. Y. (2006). Comparison of shock transmission and forearm electromyography between experienced and recreational tennis players during backhand strokes. Clinical Journal of Sport Medicine, 16(2), 129-135. doi:10.1097/ 00042752-200603000-00008

Wu, J. Z., Dong, R. G., Warren, C. M., Welcome, D. E., \& McDowell, T. W. (2014). Analysis of the effects of surface stiffness on the contact interaction between a finger and a cylindrical handle using a three-dimensional hybrid model. Medical Engineering \& Physics, 36(7), 831-841. doi:10.1016/j.medengphy.2014.03.007 